\title{
Isotropic-nematic interface in suspensions of hard rods: Mean-field properties and capillary waves
}

\author{
S. Wolfsheimer, ${ }^{1, *}$ C. Tanase, ${ }^{2}$ K. Shundyak, ${ }^{3}$ R. van Roij, ${ }^{2}$ and T. Schilling ${ }^{1}$ \\ ${ }^{1}$ Institut für Physik, Johannes Gutenberg-Universität, D-55099 Mainz, Staudinger Weg 7, Germany \\ ${ }^{2}$ Institute for Theoretical Physics, Utrecht University, Leuvenlaan 43508 TD Utrecht, The Netherlands \\ ${ }^{3}$ Instituut-Lorentz, for Theoretical Physics, Niels Bohrweg 2, Leiden, NL-2333 CA, The Netherlands
}

(Received 7 December 2005; published 9 June 2006)

\begin{abstract}
We present a study of the isotropic-nematic interface in a system of hard spherocylinders. First we compare results from Monte Carlo simulations and Onsager density functional theory for the interfacial profiles of the orientational order parameter and the density. Those interfacial properties that are not affected by capillary waves are in good agreement, despite the fact that Onsager theory overestimates the coexistence densities. Then we show results of a Monte Carlo study of the capillary waves of the interface. In agreement with recent theoretical investigations [Elgeti and Schmid, Eur. Phys. J. E 18, 407 (2005)] we find a strongly anisotropic capillary wave spectrum. For the wave numbers accessed in our simulations, the spectrum is quadratic, i.e., elasticity does not play a role. We conjecture that this effect is due to the strong bending rigidity of the director field in suspensions of spherocylinders.
\end{abstract}

DOI: 10.1103/PhysRevE.73.061703

\section{INTRODUCTION}

${ }^{1}$ Anisotropic particles form liquid crystals at low enough temperatures or high enough pressures. Elongated particles, in particular, undergo a phase transition from a phase in which particle orientations and positions are disordered (isotropic phase) to a phase in which orientations are aligned and positions are disordered (nematic phase). In many materials the direction of preferred alignment of the particles (the director $\mathbf{n}$ ) can be easily manipulated with electric or magnetic fields, which means that the optical properties of these materials can be tuned. This has made liquid crystals the basis for a large range of technological devices [1].

Liquid crystals also pose fundamental questions about the role which particle anisotropy plays in phase transitions and interfacial properties. In the 1940s Onsager showed that the transition between the isotropic phase (I) and the nematic phase $(\mathrm{N})$ is of entropic nature and that it can be explained by a simple geometrical argument [2]. However, the calculation of interfacial properties in the Onsager model proved to be far more difficult than the prediction of the bulk phase transition.

\section{A. Recent work on mean-field properties of the IN interface}

Recently there have been several theoretical investigations of the IN interface [3-11]. In the framework of density functional theory (DFT) all these studies showed that the interfacial tension of hard rods is minimal in the case of parallel anchoring, i.e., when the nematic director is parallel to the plane of the interface. Also, alignment is induced in the isotropic phase close to the interface. McMullen [3] showed by means of a variational method based on a square gradient expansion for the excluded volume that the density profile is monotonic in the case of parallel alignment. This

\footnotetext{
*Present address: Institut für Theoretische Physik FriedrichHund-Platz 137077 Göttingen
}

PACS number(s): 64.70.Md, 61.20.Ja, 64.70.Ja

result was confirmed by the full numerical studies of Chen and Noolandi $[4,5]$. They also found small biaxial effects at the interface, and a value of $\gamma_{\mathrm{IN}} L D / k_{B} T=0.187 \pm 0.001$ for the interfacial tension $\gamma_{\mathrm{IN}}$, where $L$ is the length of the particle and $D$ its diameter. This value was $50 \%$ lower than the result of the variational study by McMullen. Shundyak and van Roij [10] developed a numerical method for a full description of the excluded volume based on the assumption that the biaxiality is small, as Chen and Noolandi previously suggested. This study showed indeed a small biaxiality, being thus consistent with its hypothesis. The effect of using a finer spatial grid was that an even smaller interfacial tension value $\gamma_{\mathrm{IN}} L D / k_{B} T=0.156 \pm 0.001$ was found (which agrees with recent simulational data obtained by Vink and coworkers $[12,13])$. The density and nematic order parameter profiles were monotonic as Chen and Noolandi had also observed. Only a small nonmonotonicity appeared in the density at the isotropic side in the case of normal anchoring, i.e., when the nematic director is normal to the plane of the interface $[4,5,10]$.

Allen and co-workers have performed several simulation studies of closely related systems: they compared Onsager DFT with simulations of soft rods [14] and with simulations of hard ellipsoids confined between walls [15]; further they computed the interfacial tension of the IN interface in hard and soft ellipsoids from the pressure tensor profile [16], and studied capillary waves on the IN interface of repulsive ellipsoids [17]. In these studies they found that the interfacial tension agrees well with Onsager DFT, while the absolute coexistence densities are overestimated by theory.

In another simulation study nematic wetting films between a hard wall and an isotropic fluid of hard rods have been observed [18]. So far, however, the recent mean-field predictions on the structure and tension of the IN interface in long cylindrical rods have not been tested against computer simulations.

If one sets out to compare mean-field results to computer simulations, the role of fluctuations needs to be considered. In the following section we briefly summarize the concept of 
capillary waves, and in Sec. I C we discuss how we compare mean-field "intrinsic" profiles to our simulation results.

\section{B. Capillary waves}

At finite temperature, an interface is never planar because of thermal fluctuations ("capillary waves"). As each deviation from the planar shape costs energy, the capillary wave spectrum is determined by the balance between thermal energy and interfacial tension (and other contributions to the free energy cost of the interface, such as bending rigidity.) In the simplest case, the capillary wave spectrum is governed by the free energy cost of the interfacial area, which is added due to the undulations. Assuming that the interface position can be parametrized by a function $h(x, y)$, and that the local distortions are small, the wave spectrum is predicted to be

$$
\left\langle|h(\mathbf{q})|^{2}\right\rangle=\frac{k_{B} T}{\gamma q^{2}},
$$

where $h(\mathbf{q})$ is the Fourier transform of the local position $h(x, y)$ of the fluctuating surface, $\mathbf{q}$ is the wave vector, and $\gamma$ is the interfacial tension. Note that this expression cannot hold for $q \rightarrow 0$, because the amplitude would diverge. In real systems, gravity or finite system size, for example, introduce a cutoff at small $q$. For an introduction to the subject of capillary waves see for example the book by Safran [19].

However, the capillary wave spectrum of the IN-interface cannot be described in this simple manner. Liquid crystals exhibit long-range elastic interactions, which keep the IN interface anisotropic up to very large length scales. (The interface becomes isotropic only on scales larger than the length over which deformation of the director field costs less energy than $k_{B} T$.) Also, the interfacial tension $\gamma_{\mathrm{IN}}$ is not a constant, but a function of the angle $\phi$ between the director and the interface. Therefore, fluctuations parallel to the director will produce different free energy costs than fluctuations perpendicular to the director. This separates the IN interface from most other interfaces for which capillary waves have been studied.

Recently there have been several theoretical studies on waves on the IN interface [20-22]. In particular, the IN capillary wave spectrum has been analyzed by Elgeti and Schmid within Frank elastic theory and Landau-de Gennes theory [22]. For the Landau-de Gennes approach their study shows that

$$
\frac{k_{B} T / \gamma_{\mathrm{IN}}^{\|}}{\left\langle\left. h(\mathbf{q})\right|^{2}\right\rangle}=q^{2}+\hat{q}_{y}^{2}\left[b q^{3}+O\left(q^{4}\right)\right],
$$

where $\gamma_{\mathrm{IN}}^{\|}$is the interfacial tension for in-plane alignment (i.e., $\phi=0$ ), $\hat{q}_{y}$ is the component of the unit vector $\mathbf{q} / q$ in the direction of the bulk director, and $b$ is a constant, which contains information on the elasticity of the director field and the anisotropy of the interfacial tension. (A more detailed expression can be found in the article by Elgeti and Schmid [22].) Thus, the spectrum is isotropic in the leading $q^{2}$ term, but shows a strong anisotropy in the next-order $q^{3}$ term. An anisotropic contribution to the $q^{2}$ term, which one would expect to find because of the anisotropic interfacial tension, is removed by the elastic interactions. Both the theoretical work and the computer simulation on repulsive ellipsoids by Akino, Schmid, and Allen [17] show that the amplitudes of the waves are largest in the direction perpendicular to the director. Here, we compare these results to the hardspherocylinder case.

\section{Comparing DFT and simulation}

The IN transition in rods is of first order and it increases in strength with increasing aspect ratio. For large aspect ratios the only relevant fluctuations in a finite simulation box are therefore capillary waves. For the further discussion, we assume that there is an intrinsic mean-field interface, which is broadened by capillary waves in a manner that is statistically uncorrelated to the properties of the "intrinsic" interface. This concept has been under debate, because it clearly fails for short wavelengths, i.e., wavelengths of the order of the size of the particles [23-27]. However, it has proven to be successful in studies for which the short-wavelength limit was not relevant (see, for example, [28-30]). Here, we are interested in a comparison of simulations to Onsager theory and Landau-de Gennes theory, i.e., we are not studying molecular details and therefore we assume the intrinsic profile concept to be valid.

We test which effect fluctuations have on our DFT results, by convoluting the intrinsic DFT profile $\rho^{\mathrm{DFT}}(z)$ with a simple ansatz for the capillary wave spectrum

$$
P(h)=\frac{1}{\sqrt{2 \pi s^{2}}} \exp \left(-\frac{h^{2}}{2 s^{2}}\right),
$$

where $h$ is the local height of the interface, $s^{2}$ is the local mean-square displacement of the interfacial height,

$$
s^{2}=\frac{k_{B} T}{2 \pi \gamma} \ln \left(\frac{q_{\max }}{q_{\min }}\right),
$$

$q_{\max }$ is the length of the maximum wave vector (given by the particle diameter), and $q_{\min }$ is the length of the minimum wave vector (given by the size of the box). The estimated apparent profile then becomes

$$
\rho^{\mathrm{est}}(z)=\int_{-\infty}^{\infty} d h \rho^{\mathrm{DFT}}(z-h) P(h) .
$$

This ansatz for the capillary wave spectrum certainly does not capture the specific properties of the IN interface described in the previous section. But we will show in Sec. IV that it suffices to test whether a quantity can be compared between DFT and simulation or not.

The paper is structured as follows. First we introduce the model and the simulation techniques. Then we discuss the interfacial profiles and compare them to DFT results. In Sec. IV we present the analysis of the capillary wave spectrum and in the last section we summarize.

\section{MODEL AND ORDER PARAMETERS}

\section{A. DFT}

The basic ingredient of density functional theory is the grand potential $\Omega[\rho]$ given as a functional of the one-particle 
distribution $\rho(\mathbf{r}, \mathbf{u})$, with $\mathbf{r}$ the center of mass of a rod and $\mathbf{u}$ the unit vector of the long axis of a rod:

$$
\begin{aligned}
\beta \Omega[\rho]= & \int d \mathbf{r} d \mathbf{u} \rho(\mathbf{r}, \mathbf{u})\left\{\ln \left[\rho(\mathbf{r}, \mathbf{u}) L^{2} D\right]-1-\beta \mu\right\} \\
& -\frac{1}{2} \int d \mathbf{r} d \mathbf{u} d \mathbf{r}^{\prime} d \mathbf{u}^{\prime} f\left(\mathbf{r}, \mathbf{u} ; \mathbf{r}^{\prime}, \mathbf{u}^{\prime}\right) \rho(\mathbf{r}, \mathbf{u}) \rho\left(\mathbf{r}^{\prime}, \mathbf{u}^{\prime}\right),
\end{aligned}
$$

where $\beta=1 / k_{B} T$, and $f$ is the Mayer function which equals -1 if the rods overlap and 0 otherwise [2]. Here we focus on long hard spherocylinders with a length $L$ much larger than the diameter $D$. In the limit $D / L \rightarrow 0$, in which we perform the DFT calculations, Onsager's second virial functional is expected to be accurate $[2,5,10,11]$. For a given chemical potential $\mu$ the equilibrium density minimizes the functional, and the minimum condition $\delta \Omega[\rho] / \delta \rho(\mathbf{r}, \mathbf{u})=0$ gives rise to a nonlinear integral equation for the equilibrium profile $\rho(\mathbf{r}, \mathbf{u})$ :

$$
\ln \left[\rho(\mathbf{r}, \mathbf{u}) L^{2} D\right]-\int d \mathbf{r}^{\prime} d \mathbf{u}^{\prime} f\left(\mathbf{r}, \mathbf{u} ; \mathbf{r}^{\prime}, \mathbf{u}^{\prime}\right) \rho\left(\mathbf{r}^{\prime}, \mathbf{u}^{\prime}\right)=\beta \mu .
$$

The minimum value of the functional, which is obtained after insertion of the equilibrium profile into the functional, gives the equilibrium grand potential $-p V$ for a bulk system of volume $V$ at pressure $p$ and $-p V+\gamma A$ for a system with a planar interfacial area $A$ (with $\gamma$ the interfacial tension). In the latter geometry we assume that the distribution is independent of the in-plane coordinates $x$ and $y$, such that the Euler-Lagrange equation is to be solved for $\rho(z, \theta, \varphi)$, with $z$ the Cartesian coordinate perpendicular to the interface and $\theta$ and $\varphi$ the polar and azimuthal angles of $\mathbf{u}$ with respect to the nematic director far from the interface, respectively. Here we impose boundary conditions such that the coexisting isotropic and nematic bulk phases are obtained at $|z| \rightarrow \infty$. From the equilibrium profile one extracts $[4,5,10]$ the total density and the order parameter profile as $\rho(z)=\int d \mathbf{u} \rho(z, \mathbf{u})$ and $S(z)=\int d \mathbf{u} P_{2}(\mathbf{n} \cdot \mathbf{u}) \rho(z, \mathbf{u}) / \rho(z)$, respectively, with $P_{2}(x)=\left(3 x^{2}-1\right) / 2$ the second Legendre polynomial. The biaxiality profile is given by $\alpha(z)$ $=(3 / 2) \int d \mathbf{u} \rho(z, \mathbf{u}) \sin ^{2} \theta \cos (2 \varphi) / \rho(z)$. We present the numerical results of the corresponding profiles in Figs. 1-3. An accurate numerical method [10] gives for the surface tension $\gamma_{\|}^{\mathrm{DFT}} L D / k_{B} T=0.156 \pm 0.001$ at parallel anchoring, and $\gamma_{\perp}^{\mathrm{DFT}} L D / k_{B} T=0.265 \pm 0.001$ at normal anchoring.

\section{B. Simulations}

As in the Onsager model, we consider spherocylinders, each consisting of a cylinder of length $L$ and diameter $D$ capped by two hemispheres of diameter $D$. The interaction between two particles $i$ and $j$ is given by a pair potential of the form

$$
v_{i j}(r)=\left\{\begin{array}{cc}
\infty & r<D, \\
0 & \text { otherwise, }
\end{array}\right.
$$

where $r$ is the distance between the cylinder axes.

To investigate the IN transition, the density and the average rod alignment are used as order parameters. We

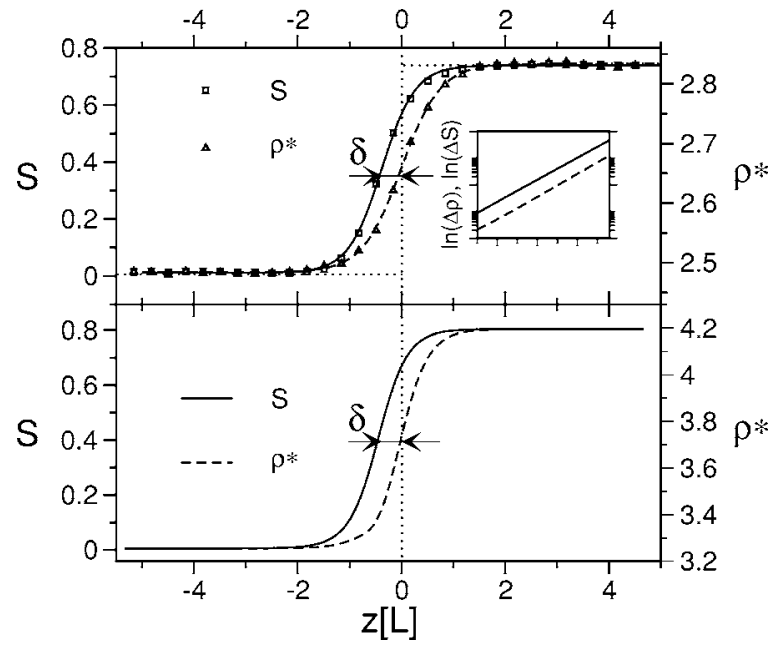

FIG. 1. Density and order parameter profiles for parallel anchoring. Upper graph: Simulation results of the reduced density $\rho^{*}$ (triangles) and the nematic order parameter $S$ (squares). Dashed and solid lines are tanh fits, which serve to guide the eye. The bulk values of the density according to simulations in the grandcanonical ensemble [13] are indicated by a dotted line. The shift $\delta$ between the inflection points of the profiles comes out clearly. Inset: Decay of the density and the order parameter to their isotropic bulk values on a logarithmic scale. Both quantities have the same decay length. Lower graph: Results from DFT calculations for density (dashed line) and nematic order parameter (solid line) profiles. Note that Onsager DFT overestimates the coexistence densities, while the shift $\delta$ between the interfaces agrees well. Differences between DFT and simulations due to fluctuations are discussed in the text.

denote the density as the dimensionless quantity $\rho^{\star}$ $=(\pi / 4) L^{2} D N / V$ where $N$ is the number of particles and $V$ is the volume of the simulation box. The average alignment is defined in terms of the orientational traceless tensor $\mathbf{Q}$ with the elements

$$
Q_{\alpha \beta}=\frac{1}{2 N} \sum_{i=1}^{N}\left(3 u_{i \alpha} u_{i \beta}-\delta_{\alpha \beta}\right)
$$

where $u_{i \alpha}$ is the $\alpha$ component $(\alpha=x, y, z)$ of the unit vector along the axis of particle $i$ and $\delta_{\alpha \beta}$ the Kronecker delta. Diagonalization yields three eigenvalues,

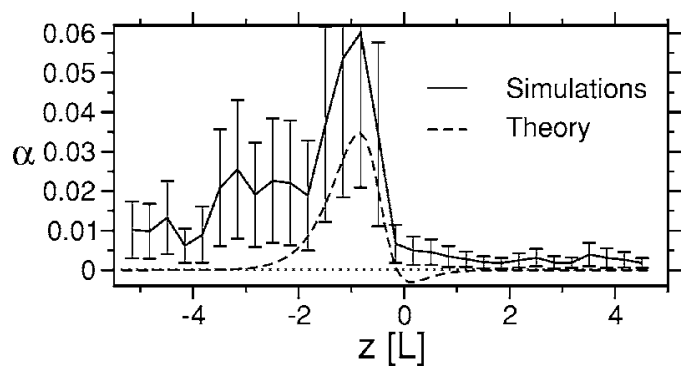

FIG. 2. Biaxiality profile from simulations (solid line) and DFT calculations (dashed line) for parallel anchoring. 


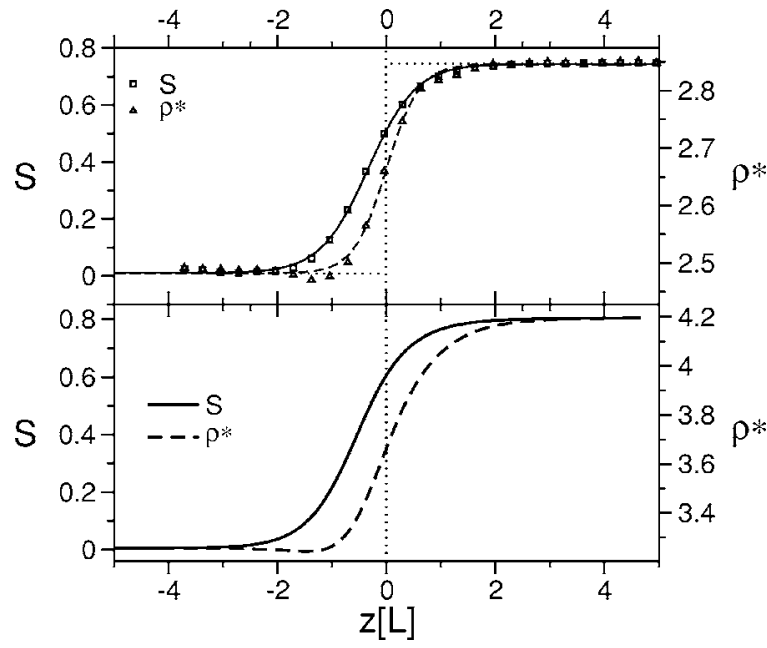

FIG. 3. Density and order parameter profiles for normal anchoring. Upper graph: Reduced density $\rho^{*}$ (triangles) and nematic order parameter $S$ (squares). Dashed and solid lines are tanh fits, which serve to guide the eye. The bulk values of the density according to simulations in the grand-canonical ensemble [13] are indicated by a dotted line. Lower graph: DFT calculations for density (dashed line) and order parameter (solid line) profiles. Both in simulation and theory, the density profile shows a small nonmonotonicity on the isotropic side.

$$
S, \quad-(S-\alpha) / 2, \quad-(S+\alpha) / 2,
$$

where $S$ is the nematic order parameter and $\alpha$ is the biaxiality, which is equivalent to the definition used in the DFT calculations.

\section{SIMULATION METHOD}

Simulations were performed in the canonical ensemble using an elongated box with periodic boundary conditions and dimensions $L_{x} \times L_{y} \times L_{z}=(10 \times 10 \times 20) L^{3}$. By rotational and translational Monte Carlo (MC) moves phase space was accessed. The aspect ratio of the rods was $L / D=15$. The choice of $L / D=15$ is motivated by the following requirements. On the one hand, the IN transition becomes weaker with decreasing $L / D$. At short $L / D$ fluctuations in the bulk phases come into play, making the comparison with DFT very difficult. On the other hand the CPU time required to detect an overlap between two rods increases with $L / D$. Thus a compromise has to be made. In a previous study on ellipsoids, $L / D=15$ had been chosen [17]. We used the same to be able to compare our results to this study.

The system was prepared in isotropic-nematic coexistence and the interfacial planes were located in the $(x, y)$ plane. Due to the periodic boundary conditions at least two interfaces were required. The overall density was chosen between the coexistence values, which we had previously determined by grand canonical simulations [13]. With this geometry about $10^{5}$ particles were required. Parallel interfacial anchoring as well as normal anchoring was investigated.

Normal anchoring is metastable; therefore the director would rotate towards a parallel orientation during a sufficiently long simulation. The time scale necessary for such a rotation is given by the time it takes to cross the free-energy barrier for the creation of a defect containing a reoriented region. As the system is relatively stiff with respect to director field bending, we expect this barrier to be rather high. During our simulation the mean $z$ component of the director decreased by less than half a percent, while the fluctuations around this value were much larger than that. We have produced some data sets using a constraint on the orientation of the director, as it was introduced by Allen and co-workers [31]. However, the results did not differ from the ones obtained in unconstraint simulations.

After the equilibration run of about $10^{7} \mathrm{MC}$ sweeps (i.e., moves per particle) the values of the density and the orientational order parameter far away from the interface did not change anymore. The bulk values were reached in clear plateaus and they were in agreement with the results from the grand canonical simulations. These conditions were used as evidence for an equilibrated state. For data production about $1.5 \times 10^{6} \mathrm{MC}$ sweeps were performed.

\section{RESULTS}

\section{A. Interfacial profiles}

To investigate the interfacial profiles, the box was divided into 60 slices in the $z$ direction, and the density, the nematic order parameter, and the biaxiality order parameter were sampled in each slice. By averaging over $3 \times 10^{5}$ profiles the statistical error on the density, which is much larger than the error on the order parameter, became reasonably small and a clear biaxiality signal could be obtained.

Note, that the total average volume of nematic phase is conserved, but that the center of the nematic layer can shift along the $z$ direction without creating any energetic or entropic costs. To account for the freedom of the system to shift in the $z$ direction, the profiles had to be centered before sampling. We shifted them such that their centers of mass lay in the middle of the simulation box. (Fluctuations of the total amount of nematic phase lead to broadening of the averaged profiles [32,33]. This effect will be discussed in Sec. IV B.) The resulting profiles for parallel anchoring from theory as well as simulation are plotted in Figs. 1 and 2.

Figures 1 and 2 show that the interface induces both nematic order and biaxiality on the isotropic side near the interface. This is in agreement with DFT calculations. The absolute values of the densities, however, differ considerably. This effect has been observed in similar systems by Allen and co-workers $[15,14]$. The induced nematic order on the isotropic side is reflected in the shift between the inflection points of the density profile and the order parameter profile, $\delta=0.38(0) L$. DFT gives a similar result, $\delta=0.45 L$ [34]. Also in computer simulations of soft spherocylinders $\delta$ $=0.37(4) L$ was found [14]. For macroscopic system sizes, this quantity is not a constant because it is affected by capillary waves. However, the profiles are almost symmetric with respect to their inflection points. Therefore convolution with a symmetric capillary wave spectrum Eq. (2) will hardly shift the inflection points and thus will not lead to changes in $\delta$ unless very large systems are considered. We tested this both by changing the size of the simulation box 
and by computing the apparent profiles $\rho^{\text {est }}(z)$ and $S^{\text {est }}(z)$ from the DFT profiles. The changes in $\delta$ were of the order of $10 \%$.

DFT also predicts that the density and order parameter profiles should decay on the same length scale, because the correlation length $\xi$ is the only characteristic length scale for these decays. The inset of Fig. 1 shows simulation results for both profiles on a logarithmic scale revealing agreement with the theoretical prediction. (For the system size used in the simulation, this property is not affected by capillary waves, because it is measured far away from the interface.)

Figure 3 shows the profiles for normal anchoring. Again there is good agreement between simulation and theory. In particular, both show a weakly nonmonotonic behavior of the density profile on the isotropic side. For increasing system sizes this dip is smoothed out by interfacial broadening.

The present simulations confirm the theoretical claims $[5,10,11]$ that, for the homeotropic (biaxial) IN interface, the density and order parameter profiles are monotonic and that the interfacial biaxiality is small. For the uniaxially symmetric IN interface the simulations confirm the theoretically predicted small nonmonotonic feature in the density profile.

\section{B. Blocking analysis}

In order to analyze capillary waves the interfacial position has to be extracted from the simulation data. This requires the choice of a definition of what constitutes the interface. Different choices of definition can lead to differences in the capillary wave spectra, in particular for short wavelengths. The simplest approach is to cut the system into blocks of length $L_{z}$ and cross section area $B \times B$ and to compute the Gibbs dividing surface for each block. However, the smaller the block, the larger are the fluctuations of the densities of the coexisting phases around their bulk values. This problem is circumvented by the method we describe below and similar methods $[17,30]$ which are based on sampling the differences between local profiles for each block in a configuration and the overall profile of the same configuration. These methods do not take into account the molecular details of the interface and are therefore not useful for studies of the capillary wave spectrum at short length scales. If one is interested in these, the pivot particle method by Tarazona and Chacón [23] can be used, which proceeds via a different route: First particles that form the outmost layer of one phase are identified. Then the minimal surface through the positions of these particles is constructed. Iteratively, more particles from the same phase which lie close to this surface are added and a new minimal surface is constructed until all surface particles are incorporated. This method has shown to produce considerable differences in capillary wave spectra at short wavelengths, and it would be worthwhile applying it to the IN interface. However, as we are interested in a comparison with Landau-de Gennes theory, a continuum theory, we use a method that is less accurate on the molecular level.

In order to analyze the capillary waves, we used the following procedure. We first determined the average plane of the interface (averaged over the whole box cross section) and then measured undulations around this reference plane. It is,

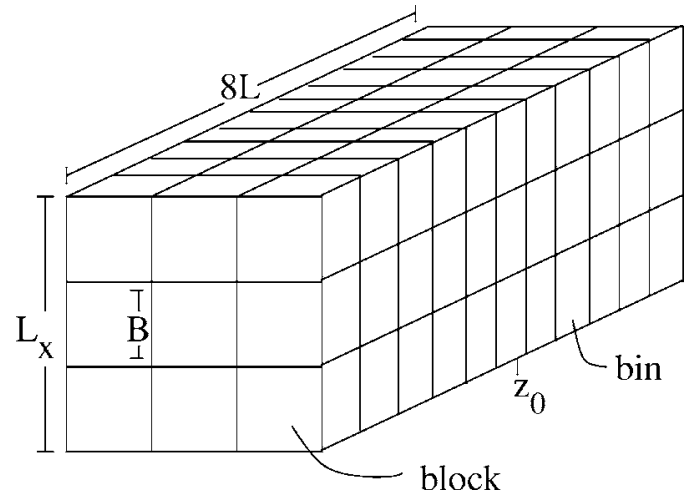

FIG. 4. Sketch of the blocking scheme. Details can be found in the text.

however, not sensible to determine a reference plane for each individual system snapshot, as the position of the averaged profile is itself a fluctuating quantity. Instead we used averages over $10^{4} \mathrm{MC}$ sweeps to determine stable reference planes. For each snapshot, we shifted the system such that its center of mass was in the center of the box. Then the box was divided into two parts, each containing one of the two interfaces. The interfaces were then investigated separately. In order to determine the reference plane of each interface we fitted the data with

$$
S(z)=\frac{1}{2}\left(S_{N}+S_{I}\right)+\frac{1}{2}\left(S_{N}-S_{I}\right) \tanh \left(\frac{z-z_{0}}{w_{S}}\right)
$$

where $S_{N}$ and $S_{I}$ are the bulk nematic and isotropic order parameters, respectively. The width $w_{S}$ and the position of the point of inflection $z_{0}$ are fit parameters. Obviously, the profiles will not be perfect hyperbolic tangent functions, because of the asymmetry in orientational order between the two phases. However, the fit only serves to determine an average interfacial position as a basis for the following analysis. As sketched in Fig. 4, the box was then divided into square blocks in the $(x, y)$ plane with side lengths $B$ varying from $L_{x} / 7$ to $L_{x}$. (Note that even the smallest block has a side length that is larger than a particle length. Thus we are far away from the molecular diameter $D$.) Each block was then divided into 25 bins in the interval $\left[z_{0}-4 L, z_{0}+4 L\right]$ in the $z$ direction. The profiles that we measured in these subsystems were again fitted by the tanh function Eq. (3) in order to find their points of inflection. All subsystem profiles for a given block size $B$ were centered at their points of inflection. Then $\mathbf{Q}$ was averaged over all profiles and the average profiles were fitted by Eq. (3) again in order to sample the squared interfacial width $w_{S}^{2}$ and the interfacial positions $z\left(x_{0}, y_{0}\right)$ (where $x_{0}, y_{0}$ denotes the position of the block center). 255 system snapshots, i.e., 510 snapshots of interfaces, have been used for the blocking analysis. The smallest subsystem contained about 7500 particles, which was sufficient to find a clear order parameter signal.

In order to make sure that the snapshots were decorrelated, we checked the autocorrelation functions of $w_{S}^{2}$ and $z$ for various block sizes. We also ruled out possible effects due to the finite bin size by comparing results for different 


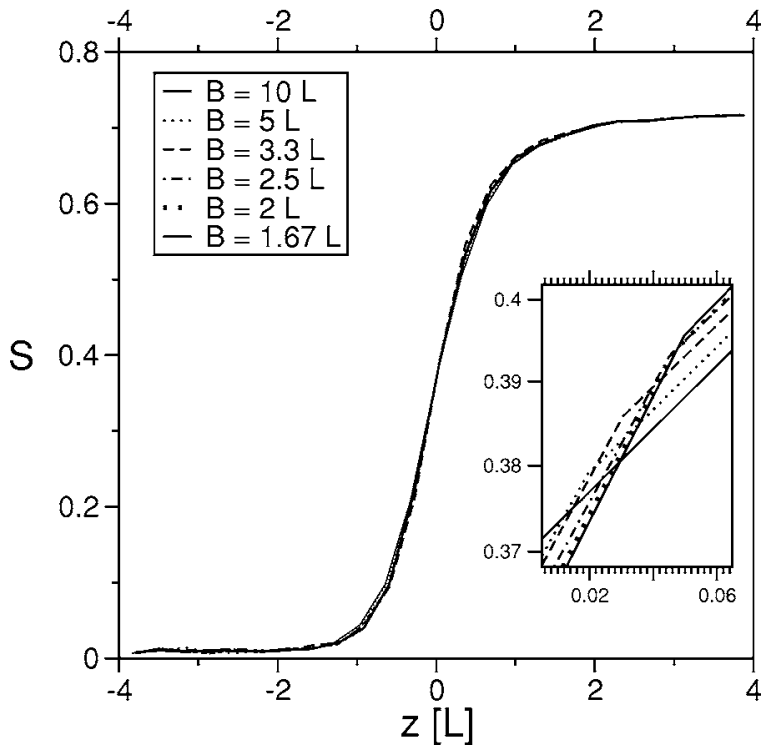

FIG. 5. Interfacial profiles for various block sizes. The inset shows a closeup view with a logarithmic scale of the profile near the center.

discretization. The size of a bin is determined by the following compromise: On the one hand a small bin size provides a good resolution of the profile, but on the other hand the computation of order parameter average requires at least 100 particles. For bin sizes between $10 D$ and $20 D$ the interfacial width as well as the computed interfacial tension remained constant within the numerical accuracy.

Figure 5 shows the averaged order parameter profiles for various block sizes. If a capillary wave spectrum is of the form of Eq. (1) the interfacial width behaves as $w_{S} \propto \sqrt{\ln (B)}$ [33]. Figure 6 shows $w_{S}^{2}$ vs $\ln (B)$ for parallel and normal anchoring. For parallel anchoring we find the predicted linear dependence. For normal anchoring there might be deviations. However, the accuracy of the simulations does not allow for a more detailed statement. The error bars have been determined by the jackknife method [35].

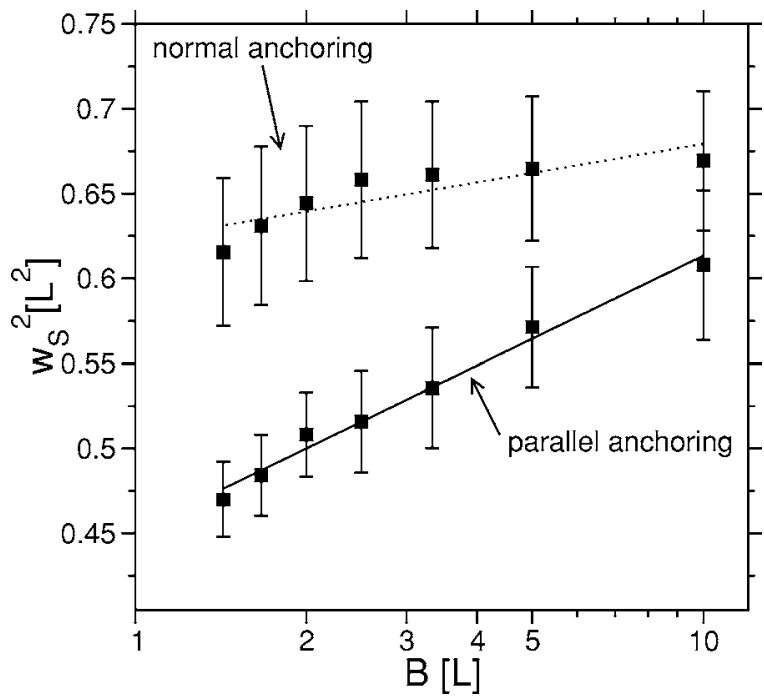

FIG. 6. Squared interfacial width $w_{S}^{2}$ versus $\ln (B)$ for parallel anchoring (solid line) and normal anchoring (dotted line).

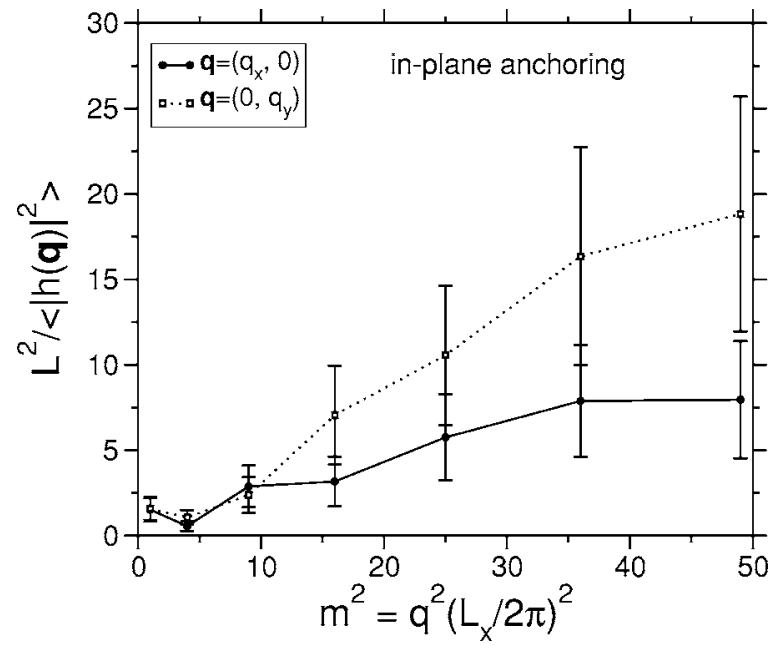

FIG. 7. Inverse mean-squared Fourier components of the interface position versus wave number squared $\left[m=q\left(L_{x} / 2 \pi\right)\right]$ for parallel anchoring.

\section{Analysis of the capillary wave spectrum}

A similar analysis as described in the previous section was used in order to measure the capillary wave spectrum. For blocks of size $L_{x} / 14 \times L_{y}$, we sampled the distances between the points of inflection of the local profiles and the point of inflection of the reference profile. In order to test the stability of our results, we also applied the method described by Akino and co-workers [17]. The accuracy was less good, but the results remained the same. Further we checked that the distribution of interfacial heights $P(h)$ was Gaussian, indicating that the system was properly thermalized. Correlations between the undulations decayed within $10^{4} \mathrm{MC}$ sweeps for the larger values of $q$ and $5 \times 10^{4}$ for the smallest value. Hence we are confident that the snapshots over which we averaged were sufficiently decorrelated.

Figures 7 and 8 show $L^{2} /\left\langle|h(\mathbf{q})|^{2}\right\rangle$ vs wave number squared for parallel and normal anchoring. (We have plotted $\mathbf{m}=\mathbf{q}\left(L_{x} / 2 \pi\right)$ instead of $q$ to facilitate direct comparison to

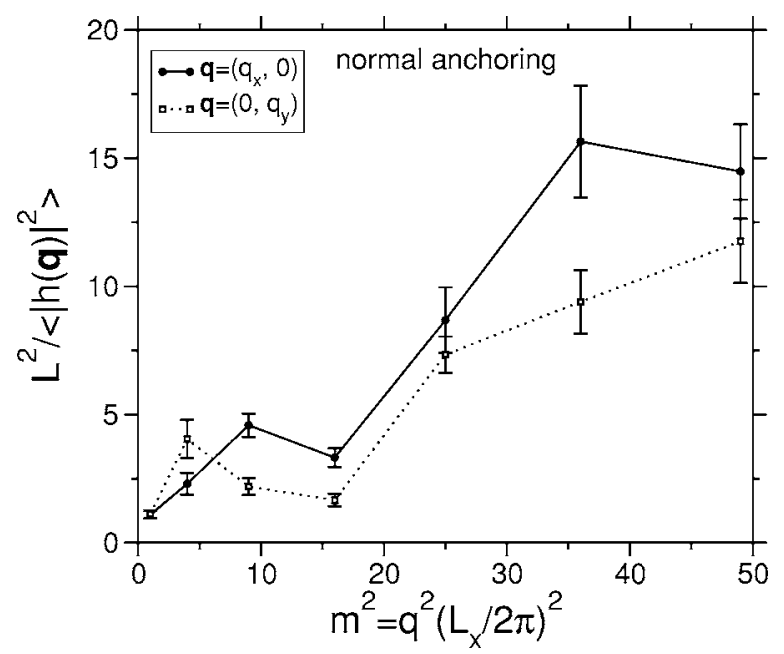

FIG. 8. Inverse mean-squared Fourier components of the interface position versus wave number squared for normal anchoring. 


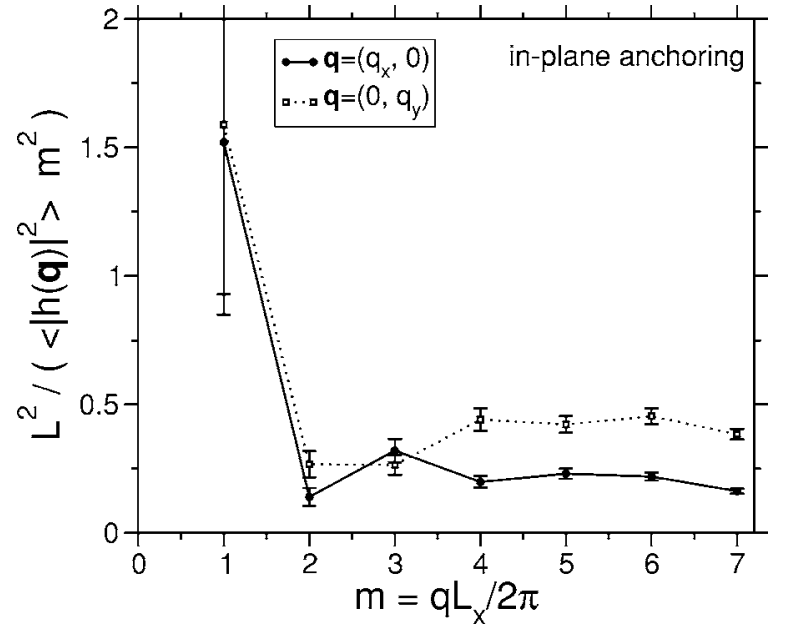

FIG. 9. $L^{2} /\left[\left\langle|h(\mathbf{q})|^{2}\right\rangle m^{2}\right]$. For $m>2$ amplitudes are proportional to $1 / q^{2}$. The anisotropy of the interfacial tension shows already on the $q^{2}$ level.

the results of Akino and co-authors [17]). Again the error bars have been determined by the jackknife method. In the case of parallel anchoring the director was oriented along the $y$ axis. As expected, the spectrum is anisotropic for parallel anchoring and isotropic for normal anchoring. For parallel anchoring, we find in agreement with the theory [22] that fluctuations parallel to the director (dotted line) are smaller than fluctuations perpendicular to the director (solid line).

The magnitude of the fluctuations compares well with the results Akino and coauthors have obtained for ellipsoids [17]. However, their results deviate from the $q^{2}$ dependence already for $m^{2} \approx 10$. To check whether there are contributions of higher order than $q^{2}$, we have plotted $L^{2} /\left[\left\langle|h(\mathbf{q})|^{2}\right\rangle m^{2}\right]$ versus wave number for parallel anchoring in Fig. 9. For $m$ $>2$, both components are almost constant. (The large values for very small wave numbers are due to the finite system size.) Parallel to the director, there might be small deviations from the $q^{2}$ dependence, but the results are not accurate enough to allow for detailed conclusions. Additionally we find that there is a difference in the absolute value $L^{2} /\left[\left\langle|h(\mathbf{q})|^{2}\right\rangle m^{2}\right]$ between parallel and perpendicular contributions. This means that for the regime of wave numbers studied in this work, the anisotropy of the interfacial tension does enter already on the $q^{2}$ level.

Figure 10 shows a sketch of capillary waves in two extreme cases. In the case on the left, parallel anchoring is so strong that the particles' orientations have to follow the shape of the interface. The anisotropy of the spectrum is then dominated by contributions from bending elasticity. In the case on the right, the bending rigidity is so strong, that the anisotropy of the spectrum is dominated by contributions from the anisotropy of the interfacial tension. From the fact that we do not observe contributions due to bending, but strong contributions due to interfacial tension anisotropy, we conclude that spherocylinders fall into the category sketched on the right.
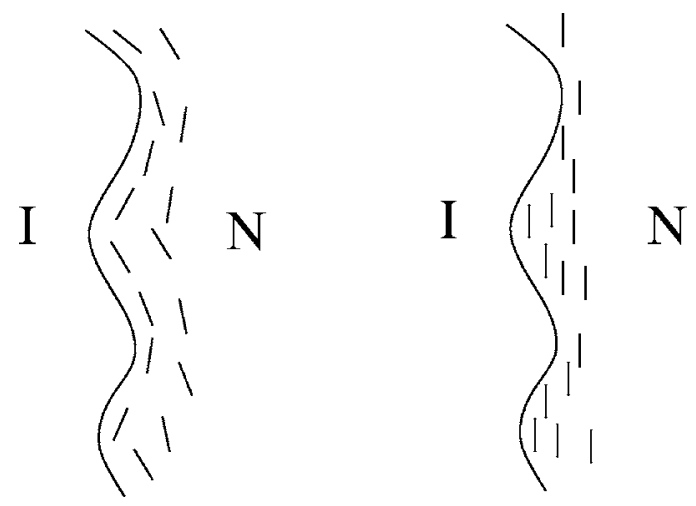

FIG. 10. Sketch of capillary waves. Left: Strong interfacial anchoring: anisotropy of the spectrum is dominated by elasticity. Right: Strong bending rigidity: anisotropy of the spectrum is dominated by the anisotropy of the interfacial tension.

\section{DISCUSSION AND SUMMARY}

We have presented a comparative study of computer simulations and Onsager DFT for the isotropic-nematic interface in hard spherocylinders. Although Onsager theory overestimates the isotropic-nematic coexistence densities, interfacial properties such as the shift between the density profile and the nematic order parameter profile are in agreement. This observation accords with the studies by Allen and coworkers on ellipsoids [15] and soft spherocylinders [14].

Our simulations confirm that, for the case of in-plane orientation of the director at the interface, the density and order parameter profiles are monotonic and that the interfacial biaxiality is small. For the case of normal orientation the simulations agree with the theoretically predicted small nonmonotonic feature in the density profile.

The capillary wave spectrum of the interface (in the case of parallel anchoring) is anisotropic, as expected. In contrast to the case of ellipsoids, we find that $L^{2} /\left\langle|h(\mathbf{q})|^{2}\right\rangle \propto q^{2}$ for the entire range of wave vectors which could be accessed in this study. Also we observe anisotropic contributions already on the $q^{2}$ level. We interpret both facts as signals of a very stiff director field, which prohibits bending along the fluctuating interface.

\section{ACKNOWLEDGMENTS}

We thank the Deutsche Forschungsgemeinschaft (DFG) for support Contract No. (TR6/D5) and K. Binder, R. Vink, P. van der Schoot, and J. Horbach for helpful suggestions. T.S. is supported by the Emmy Noether program of the DFG and by the MWFZ. Allocation of computer time on the JUMP at the Forschungszentrum Jülich is gratefully acknowledged. This work is part of the research program of the "Stichting voor Fundamenteel Onderzoek der Materie (FOM)," which is financially supported by the "Nederlandse organisatie voor Wetenschappelijk Onderzoek (NWO)." 
[1] P. G. de Gennes and J. Prost, The Physics of Liquid Crystals (Clarendon Press, Oxford, 1993).

[2] L. Onsager, Ann. N.Y. Acad. Sci. 51, 627 (1949).

[3] W. E. McMullen, Phys. Rev. A 38, 6384 (1988).

[4] Z. Y. Chen and J. Noolandi, Phys. Rev. A 45, 2389 (1992).

[5] Z. Y. Chen, Phys. Rev. E 47, 3765 (1993).

[6] D. L. Koch and O. G. Harlen, Macromolecules 32, 219 (1999).

[7] P. van der Schoot, J. Phys. Chem. B 103, 8804 (1999).

[8] E. Velasco, L. Mederos, and D. E. Sullivan, Phys. Rev. E 66, 021708 (2002).

[9] M. P. Allen, Chem. Phys. Lett. 331, 513 (2000).

[10] K. Shundyak and R. van Roij, J. Phys.: Condens. Matter 13, 4789 (2001).

[11] K. Shundyak and R. van Roij, Phys. Rev. E 68, 061703 (2003).

[12] R. L. C. Vink and T. Schilling, Phys. Rev. E 71, 051716 (2005).

[13] R. L. C. Vink, S. Wolfsheimer, and T. Schilling J. Chem. Phys. 123, 074901 (2005).

[14] M. S. Al-Barwani and M. P. Allen, Phys. Rev. E 62, 6706 (2000).

[15] M. P. Allen, J. Chem. Phys. 112, 5447 (2000).

[16] A. J. McDonald, M. P. Allen, and F. Schmid, Phys. Rev. E 63, 010701(R) (2000).

[17] N. Akino, F. Schmid, and M. P. Allen, Phys. Rev. E 63, 041706 (2001).

[18] M. Dijkstra, R. van Roij, and R. Evans, Phys. Rev. E 63, 051703 (2001); R. van Roij, M. Dijkstra, and R. Evans, Europhys. Lett. 49, 350 (2000); M. Dijkstra and R. van Roij, J.
Phys.: Condens. Matter 17, S3507 (2005).

[19] S. A. Safran, Statistical Thermodynamics of Surfaces, Interfaces, and Membranes (Addison-Wesley Publishing Company, New York, 1994).

[20] V. Popa-Nita and T. J. Sluckin, Phys. Rev. E 66, 041703 (2002).

[21] V. Popa-Nita and P. Oswald, Phys. Rev. E 68, 061707 (2003).

[22] J. Elgeti and F. Schmid, Eur. Phys. J. E 18, 407 (2005).

[23] P. Tarazona and E. Chacón, Phys. Rev. B 70, 235407 (2004).

[24] J. Stecki, J. Chem. Phys. 109, 5002 (1998).

[25] M. Napiorkowski and S. Dietrich, Phys. Rev. E 47, 1836 (1993).

[26] K. R. Mecke and S. Dietrich, Phys. Rev. E 59, 6766 (1999).

[27] A. Robledo, C. Varea, and V. Romero-Ronchin, Physica A 177, 474 (1991).

[28] A. Werner, F. Schmid, M. Müller, and K. Binder, J. Chem. Phys. 107, 8175 (1997).

[29] A. Werner, F. Schmid, M. Müller, and K. Binder, Phys. Rev. E 59, 728 (1999).

[30] R. Vink, J. Horbach, and K. Binder, J. Chem. Phys. 122, 134905 (2005).

[31] M. P. Allen, M. A. Warren, M. R. Wilson, A. Sauron, and W. Smith, J. Chem. Phys. 105, 2850 (1996).

[32] H. L. Tepper and W. J. Briels, J. Chem. Phys. 116, 5186 (2002).

[33] K. Binder, Phys. Rev. A 25, 1699 (1982).

[34] K. Shundyak, Ph.D. dissertation, University of Utrecht, The Netherlands, 2004.

[35] B. Efron, Ann. Stat. 7, 1 (1979). 